\title{
Radiation hazards for cardiologists
}

\author{
MJ RAPHAEL, * NE TOMLINSON† \\ From the ${ }^{\star}$ National Heart Hospital and $†$ Department of Clinical Physics, Guy's Hospital, London
}

At its March 1985 meeting in Paris ${ }^{1}$ the International Commission on Radiation Protection (ICRP) heavily reinforced the recommendation that the maximum permissible dose of radiation to the lens of the eye should be $150 \mathrm{mSv}$ per year of working life. ${ }^{2}$ Is this of relevance to interventional cardiologists and what should they do about it?

In correctly set up apparatus and when the operator is wearing the standard effective (that is undamaged) protective lead apron the chief hazard to interventional cardiologists and radiologists is radiation damage to the lens of the eye leading to cataracts. ${ }^{2}$ Are critical eye dose levels likely to be reached in interventional cardiologists? The answer is not clear, particularly with modern multiangulated $x$ ray apparatus and with modern techniques such as coronary angioplasty and pacing.

In 1978 Balter et al studied the radiation dose to operators during coronary arteriography using modern multiangulated apparatus and performed from the arm. ${ }^{3}$ The eyes were considered to be the organs most at risk receiving $0.06 \mathrm{mSv}$ without the use of additional shielding and $0.019 \mathrm{mSv}$ with the special shields during angiographic examination. This group concluded that an operator could perform 29 examinations per week (mean fluoroscopy $12.7 \mathrm{~min}$ and mean cine radiography $110 \mathrm{~s}$ ) without reaching the ICRP threshold for non-designated workers (that is those who are unlikely to exceed $30 \%$ of the maximum permissible dose).

Since 1978 pacemaker implants and coronary angioplasty, with their long screening times, have become more widespread and a recent paper, ${ }^{4}$ combined with the ICRP reassertion of the maximum permissible dose to the eye, has revived the issue of what is a safe workload for the interventional cardiologist.

Jeans et al measured isodose curves produced from a modern multiangulated set and a water phantom, and used the curves to calculate dose rates for staff present in the $x$ ray room during various cardiac interventional procedures. ${ }^{4}$ This phantom neglects

Requests for reprints to Dr M J Raphael, National Heart Hospital, Westmoreland Street, London W1M 8BA. the effect on scattered radiation production and distribution of the large air volume in the lungs. The paper also makes use of a "weighted surface dose" which is not an ICRP concept. Jeans et al concluded that interventional cardiologists doing 200 cardiac catheterisations, one or two angioplasties, and 30 pacemaker implants in a year would remain within safe exposure limits. The addition of five further angioplasties, electrophysiological studies, or the use of more complex catheter ablation techniques on arrhythmia patients would increase the average dose that interventional cardiologists would receive to three tenths of the maximum permissible eye dose. When they measured actual dose levels, however, they found that these were only about one third of those calculated from the phantom study.

These measured values accord fairly well with those published by Dash and Leaman in 1984 which were made on a rotating cradle rather than on a dedicated multiangle tube support system. ${ }^{5}$ These workers found eye lens doses of $0.175 \mathrm{mSv}$ per angioplasty procedure and rather lower $0.075 \mathrm{mSv}$ per angiogram. This suggests that a single operator could perform over 650 coronary angiographic examinations or 285 angioplasty procedures per year before reaching the $50 \mathrm{mSv}$ limit of eye dose. Dash and Leaman found that lead glasses reduce the eye dose by a factor of $35 \%$. Similar conclusions were obtained from the measurements by Balter et al. ${ }^{3}$

Under the new regulations ${ }^{6}$ any worker who is likely to exceed $30 \%$ of the maximum permissible dose is classified as a designated worker. Such workers must be rigorously monitored. If there is any possibility of their exceeding the maximum permissible dose they should be stopped; and they may be restrained by law.

Should all those who perform coronary angioplasty as well as other catheterisation procedures automatically become designated workers? Probably not, but in view of the three fold difference between the conclusions of Balter $\mathrm{et} \mathrm{al}^{3}$ and those of Jeans et $\mathrm{al}^{4}$ doses of radiation to the eyes should be measured in more at risk workers. The local radiation protection advisor should measure doses in all staff who are likely to be affected, since local variations in approach and practice may be major determinants of 
safety.

The ICRP also drew attention to reported examples of practices causing concern. These included the performance of complex radiological procedures by physicians or surgeons without training in radiology and radiation protection. Some operators may feel that the needs of the patient outweigh a future risk of radiation injury to themselves, and on occasion individuals have removed monitoring devices to avoid the identification of high dose levels. ${ }^{1}$ Such actions will be illegal in a designated worker.

Until the magnitude of the problem is known cardiologists should use the following simple measures to minimise radiation dosage to themselves.

Those performing catheterisations should be instructed in radiation safety practice before or early in their training. Simple guidelines and an understanding of the main source of irradiation to the operator should help to improve practice.

There are two sources of radiation to the operator-the main beam and radiation scatter from the main beam as it traverses the patient. The operator must never get any part of his or her body in the main beam where dose is highest. The lead shutters on the $x$ ray tube should be used to produce the smallest aperture necessary to perform the procedure. This is because the dosage to the patient and the scatter to the staff increase with the increase of area of patient irradiated. A small aperture also improves the quality of the image, which is degraded by scatter. Proper maintenance of the apparatus and quality control will also reduce radiation to both staff and patients.

Screening and cineradiography times should be kept as short as possible, and if possible the cine framing rate should be reduced ( 25 frames per second is usually adequate). The operator dose is markedly attenuated by distance, so it is worth while taking a step backwards away from the patient during cineradiography, because doses to the patient and operator during cineradiography are twenty times higher than during fluoroscopy. ${ }^{3}$

A projection which minimises scatter to the operator should be chosen. Scatter is mostly back scatter and is increased when the $x$ ray tube is placed between the patient and the operator, ${ }^{4}$ rather than beyond the patient. Lead aprons $(0.35 \mathrm{~mm}$ lead equivalent), which should always be worn, reduce the trunk dose by a factor of approximately 15 .

Operators who perform angioplasties may need to protect their eyes with a shield or spectacles made of lead glass. Even photochromic sunglasses have a considerable protective effect.

In any group of cardiologists the workload should be shared so that individuals avoid the risk of excessive doses. The Manchester group 4 suggested that about 1000 cardiac catheterisations, eight angioplasties, and 150 pacemaker implants was a sensible workload for five cardiologists who do not use special eye protection and who wish to avoid classification as designated radiation workers.

\section{References}

1 Statement from the 1985 Paris Meeting of the International Commission on Radiological Protection. $\mathrm{Br} \boldsymbol{F}$ Radiol 1985; 58: 910.

2 International Commission on Radiological Protection 77. ICRP Publication 26. Annals of the ICRP 1977; 1 (3).

3 Balter S, Sones FM Jr, Brancato R. Radiation exposure to the operator performing cardiac angiography with U-arm systems. Circulation 1978; 58: 925-32.

4 Jeans SP, Faulkner K, Love HG, Bardsley RA. An investigation of the radiation dose to staff during cardiac radiological studies. Br f Radiol 1985; 58: 419-28.

5 Dash H, Leaman DM. Operator radiation exposure during percutaneous transluminal coronary angioplasty. I Am Coll Cardiol 1984; 4: 725-8.

6 Ionising radiations regulations health and safety. Statutory Instruments 1985; 1333. London: HMSO, 1985. 